\title{
Positive solutions for a class of nonlinear Hadamard fractional differential equations with a parameter
}

\author{
Haisong Huang ${ }^{1 *}$ and Weihua Liu²
}

\section{${ }^{*}$ Correspondence:}

highmathematics@163.com

${ }^{1}$ Faculty of General Education,

Zhengzhou Technology and

Business University, Zhengzhou,

China

Full list of author information is

available at the end of the article

\section{黛 Springer}

\begin{abstract}
In this paper, we investigate a class of boundary value problem of nonlinear Hadamard fractional differential equations with a parameter. By means of the properties of the Green function and Guo-Krasnosel'skii fixed-point theorem on cones, the existence and nonexistence of positive solutions are obtained. Finally, some examples are presented to show the effectiveness of our main results.
\end{abstract}

MSC: 34B18; 26A33; 34A34

Keywords: Hadamard fractional differential equations; Positive solutions; Fixed-point theorem on cones

\section{Introduction}

Fractional differential equations have given rise to abroad attention of many researchers by the intensive development of the theory of fractional calculus itself. On the other hand, fractional differential equations can better describe many phenomena than ordinary differential equations in many diverse and widespread fields of science and engineering. For the development of fractional calculus and applications, we refer the reader to [1-10] and the references therein. For example, by employing the Avery-Henderson fixed-point theorem, $\mathrm{Li}$ [11] obtained the existence of positive solutions as considered for a fractional differential equation with $p$-Laplacian operator. In [12], existence and uniqueness results for a new class of boundary value problems of sequential fractional differential equations with nonlocal non-separated boundary conditions involving lower-order fractional derivatives were given by some standard fixed-point theorems. The existence and multiplicity of solutions or positive solutions for nonlinear boundary value problems involving fractional differential equations with kinds of boundary value conditions were studied by some wellknown fixed-point theorems, the lower and upper solutions method and the monotone iterative technique; see $[13,14]$ and the references therein. For example, the authors of [15] investigated the solutions of fractional integrodifferential equations with boundary value conditions, respectively. In [16], the existence and multiplicity of positive solutions were obtained for nonlinear Caputo fractional differential equations with integral boundary conditions. Henderson and Luca investigated the positive solutions of nonlinear boundary value problems for systems of fractional differential equations in the book [17]. In [18], by applying the fixed-point theorem due to Leggett-Williams, the authors considered the

(c) The Author(s) 2018. This article is distributed under the terms of the Creative Commons Attribution 4.0 International License (http://creativecommons.org/licenses/by/4.0/), which permits unrestricted use, distribution, and reproduction in any medium, provided you give appropriate credit to the original author(s) and the source, provide a link to the Creative Commons license, and indicate if changes were made. 
existence of positive solutions for a system of fractional multi-point boundary value problem with $p$-Laplacian operator.

In the past ten years, most of the work on the topic is based on Riemann-Liouvilleand Caputo-type fractional differential equations. Recently, more and more scholars paid attention to the boundary value problems of nonlinear Hadamard fractional differential equations [19-24]. By applying some standard fixed-point theorems, Ahmad and Ntouyas [25, 26] studied the existence and uniqueness of solutions for Hadamard-type fractional differential equations for boundary value problems and systems with integral boundary conditions, respectively. Based on standard fixed-point theorems for multivalued maps, Ahmad et al. [27] investigated the existence of solutions for fractional boundary value problems involving Hadamard-type fractional differential inclusions and integral boundary conditions. Aljoudi et al. [28] studied a nonlocal boundary value problem of Hadamard-type coupled sequential fractional differential equations supplemented with coupled strip conditions. By discussing a continuity, integrable estimation, and the asymptotic property on Mittag-Leffler functions, Li and Wang [29] investigated the existence of solutions and finite-time stability for a class of nonlinear Hadamard fractional differential equations with constant coefficient. In [30,31], the existence of positive solutions for nonlinear Hadamard fractional differential equations with four-point coupled and coupled integral boundary conditions were given by the Guo-Krasnosel'skii fixed-point theorems, respectively.

From the above review of the literature concerning fractional differential equations, most of the authors investigated only the existence of solutions or positive solutions for Hadamard fractional differential equations without considering the influence of a parameter. In this paper, we will study the influence of parameter intervals for Hadamard fractional differential equation boundary value problems. Motivated by the work mentioned above, we consider the following nonlinear Hadamard fractional differential equation with a parameter:

$$
\left(D^{\alpha} x\right)(t)+\lambda a(t) f(x(t))=0, \quad x(1)=(\delta x)(1)=(\delta x)(e)=0, \alpha \in(2,3], t \in[1, e],
$$

where $\lambda$ is a positive parameter, $D^{\alpha}$ is the left-sided Hadamard fractional derivative or$\operatorname{der} \alpha,(\delta x)(t)=t d x(t) / d t, a:(1, e) \rightarrow[0, \infty)$ and $f:[0, \infty) \rightarrow[0, \infty)$ are two continuous functions. The main aim of this paper is to investigate the above Hadamard fractional differential equation boundary value problem (1). With the help of the properties of the Green function and the Guo-Krasnosel'skii fixed-point theorem on cones, we establish the existence and nonexistence of positive solutions. At the end, we give some examples to illustrate the feasibility of our proposed theoretical results.

\section{Preliminaries}

For convenience of the reader, we present some necessary definitions and lemmas from Hadamard fractional calculus theory in this section.

Definition 2.1 ([32]) The left-sided Hadamard fractional integrals of order $\alpha \in \mathbb{R}^{+}$of the function $y(t)$ are defined by

$$
\left(I^{\alpha} y\right)(t)=\frac{1}{\Gamma(\alpha)} \int_{1}^{t}\left(\ln \frac{t}{s}\right)^{\alpha-1} y(s) \frac{d s}{s} \quad(1 \leq t \leq e),
$$

where $\Gamma(\cdot)$ is the Gamma function. 
Definition 2.2 ([32]) The left-sided Hadamard fractional derivatives of order $\alpha \in[n-$ $1, n), n \in \mathbb{Z}^{+}$, of the function $y(t)$ are defined by

$$
\left(D^{\alpha} y\right)(t)=\frac{1}{\Gamma(n-\alpha)}\left(t \frac{d}{d t}\right)^{n} \int_{1}^{t}\left(\ln \frac{t}{s}\right)^{n-\alpha+1} y(s) \frac{d s}{s} \quad(1 \leq t \leq e),
$$

where $\Gamma(\cdot)$ is the Gamma function.

Now we will give the Green function of linear equation and some properties of the Green function.

Lemma 2.1 Let $\alpha \in(0,1]$ be fixed and $y \in C[1, e]$, then the linear boundary value problem

$$
\left(D^{\alpha} x\right)(t)+y(t)=0, \quad x(1)=(\delta x)(1)=(\delta x)(e)=0, \alpha \in(2,3], t \in[1, e],
$$

has a unique solution which is given by the following integral representation of the solution:

$$
x(t)=\int_{1}^{e} G(t, s) y(s) \frac{d s}{s}, \quad t \in[1, e]
$$

where

$$
G(t, s)=\frac{1}{\Gamma(\alpha)} \begin{cases}(1-\ln s)^{\alpha-2}(\ln t)^{\alpha-1}, & 1 \leq t \leq s \leq e, \\ (1-\ln s)^{\alpha-2}(\ln t)^{\alpha-1}-(\ln (t / s))^{\alpha-1}, & 1 \leq s \leq t \leq e .\end{cases}
$$

Proof As argued in [32], the solution of Hadamard differential equation (2) can be written the following equivalent integral equations:

$$
x(t)=c_{1}(\ln t)^{\alpha-1}+c_{2}(\ln t)^{\alpha-2}+c_{3}(\ln t)^{\alpha-3}-\frac{1}{\Gamma(\alpha)} \int_{1}^{t}\left(\ln \frac{t}{s}\right)^{\alpha-1} y(s) \frac{d s}{s},
$$

where $c_{1}, c_{2}, c_{3} \in \mathbb{R}$. From the boundary condition $x(1)=0$, we have $c_{3}=0$. Furthermore, from (5), we can get

$$
(\delta x)(t)=c_{1}(\alpha-1)(\ln t)^{\alpha-2}+c_{2}(\alpha-2)(\ln t)^{\alpha-3}-\frac{\alpha-1}{\Gamma(\alpha)} \int_{1}^{t}\left(\ln \frac{t}{s}\right)^{\alpha-2} y(s) \frac{d s}{s} .
$$

From the boundary condition $(\delta x)(0)=(\delta x)(1)=0$ and $(6)$, we obtain $c_{2}=0$ and

$$
c_{1}=\frac{1}{\Gamma(\alpha)} \int_{1}^{e}(1-\ln s)^{\alpha-2} y(s) \frac{d s}{s} .
$$

Substituting (7) and $c_{2}=c_{3}=0$ into (5), we can observe

$$
\begin{aligned}
x(t) & =\frac{1}{\Gamma(\alpha)} \int_{1}^{e}(1-\ln s)^{\alpha-2}(\ln t)^{\alpha-1} y(s) \frac{d s}{s}-\frac{1}{\Gamma(\alpha)} \int_{1}^{t}\left(\ln \frac{t}{s}\right)^{\alpha-1} y(s) \frac{d s}{s} \\
& =\int_{1}^{e} G(t, s) y(s) \frac{d s}{s},
\end{aligned}
$$

which implies (3). The proof is completed. 
Lemma 2.2 Let $G(t, s)$ be defined as in (4) and $g(t)=(\ln t)^{\alpha-1}$. Then the following inequalities hold:

$$
G(t, s) \geq 0, \quad g(t) G(e, s) \leq G(t, s) \leq G(e, s), \quad \forall 1 \leq s, t \leq e .
$$

Proof For $1 \leq t \leq s \leq e$, It is easy to see that $G(t, s) \geq 0$ and $g(t) G(e, s)=G(t, s) \leq G(e, s)$.

For $1 \leq s \leq t \leq e$, we have

$$
\begin{aligned}
\Gamma(\alpha) G(t, s) & =(1-\ln s)^{\alpha-2}(\ln t)^{\alpha-1}-(\ln (t / s))^{\alpha-1} \\
& =(1-\ln s)^{\alpha-2}(\ln t)^{\alpha-1}-(1-\ln s / \ln t)^{\alpha-1}(\ln t)^{\alpha-1} \\
& \geq(1-\ln s)^{\alpha-2}(\ln t)^{\alpha-1}-(1-\ln s)^{\alpha-1}(\ln t)^{\alpha-1}=0,
\end{aligned}
$$

which implies $G(t, s) \geq 0$. Let $h(t)=(1-\ln s)^{\alpha-2}(\ln t)^{\alpha-1}-(\ln (t / s))^{\alpha-1}$ for $1 \leq t \leq e$. Then

$$
\begin{aligned}
d h(t) / d t & =(\alpha-1)\left[(1-\ln s)^{\alpha-2}(\ln t)^{\alpha-2}-(\ln (t / s))^{\alpha-2}\right] \\
& \geq(\alpha-1)(\ln t)^{\alpha-2}\left[(1-\ln s)^{\alpha-2}-(1-\ln s)^{\alpha-2}\right]=0,
\end{aligned}
$$

which implies that $h(t)$ is the monotone nondecreasing function, i.e. $G(t, s) \leq G(e, s)$. On the other hand,

$$
\begin{aligned}
\frac{G(t, s)}{G(e, s)} & =\frac{(1-\ln s)^{\alpha-2}(\ln t)^{\alpha-1}-(\ln (t / s))^{\alpha-1}}{(1-\ln s)^{\alpha-2}-(1-\ln s)^{\alpha-1}} \\
& \geq \frac{(1-\ln s)^{\alpha-2}(\ln t)^{\alpha-1}-(\ln t-\ln t \ln s)^{\alpha-1}}{(1-\ln s)^{\alpha-2}-(1-\ln s)^{\alpha-1}}=(\ln t)^{\alpha-1}=g(t),
\end{aligned}
$$

which implies $g(t) G(e, s) \leq G(t, s)$. The proof of Lemma 2.2 is completed.

Our main results are based on the following Guo-Krasnosel'skii fixed-point theorem on cones.

Lemma $2.3([33,34])$ Let $\mathcal{X}$ be a Banach space, and let $\mathcal{P} \subset \mathcal{X}$ be a cone in $\mathcal{X}$. Assume that $\Omega_{1}, \Omega_{2}$ are open subsets of $\mathcal{X}$ with $0 \in \Omega_{1} \subset \bar{\Omega}_{1} \subset \Omega_{2}$, and let $S: \mathcal{P} \rightarrow \mathcal{P}$ be a completely continuous operator such that, either

$\left(B_{1}\right)\|S w\| \leq\|w\|, w \in \mathcal{P} \cap \partial \Omega_{1}$, and $\|S w\| \geq\|w\|, w \in \mathcal{P} \cap \partial \Omega_{2}$,

or

$\left(B_{2}\right)\|S w\| \geq\|w\|, w \in \mathcal{P} \cap \partial \Omega_{1}$, and $\|S w\| \leq\|w\|, w \in \mathcal{P} \cap \partial \Omega_{2}$.

Then $S$ has a fixed point in $\mathcal{P} \cap\left(\bar{\Omega}_{2} \backslash \Omega_{1}\right)$.

Let $\mathscr{E}=C[1, e]$ be the Banach space endowed with the norm $\|x\|=\sup _{t \in[1, e]}|x(t)|$. Define the cone $\mathcal{P} \subset \mathcal{E}$ by

$$
\mathcal{P}=\{x \in \mathcal{E}: x(t) \geq g(t)\|x\|, t \in[1, e]\}, \quad g(t)=(\ln t)^{\alpha-1}
$$

Suppose that $x$ is a solution of boundary value problem (1). Then from Lemma 2.1, we obtain

$$
x(t)=\lambda \int_{1}^{e} G(t, s) a(s) f(x(s)) \frac{d s}{s}, \quad t \in[1, e] .
$$


We define an operator $S_{\lambda}: \mathcal{P} \rightarrow \mathcal{E}$ as follows:

$$
\left(S_{\lambda} x\right)(t)=\lambda \int_{1}^{e} G(t, s) a(s) f(x(s)) \frac{d s}{s}, \quad t \in[1, e]
$$

By Lemma 2.2, we have

$$
\begin{aligned}
& \left\|S_{\lambda} x\right\| \leq \lambda \int_{1}^{e} G(e, s) a(s) f(x(s)) \frac{d s}{s}, \\
& \left(S_{\lambda} x\right)(t) \geq \lambda \int_{1}^{e} g(t) G(e, s) a(s) f(x(s)) \frac{d s}{s} \geq g(t)\left\|S_{\lambda} x\right\| .
\end{aligned}
$$

Thus, $S_{\lambda}(\mathcal{P}) \subset \mathcal{P}$. Then we have the following lemma.

Lemma 2.4 $S_{\lambda}: \mathcal{P} \rightarrow \mathcal{P}$ is completely continuous.

Proof The operator $S_{\lambda}: \mathcal{P} \rightarrow \mathcal{P}$ is continuous in view of continuity of $G(t, s), a(t)$ and $f(x(t))$. By means of the Arzela-Ascoli theorem, $S_{\lambda}: \mathcal{P} \rightarrow \mathcal{P}$ is completely continuous.

\section{Main results}

In this section, we establish some sufficient conditions for the existence and nonexistence of positive solutions for boundary value problem (1).

For convenience, we denote

$$
\begin{aligned}
& F_{0}=\limsup _{x \rightarrow 0^{+}} \frac{f(x)}{x}, \quad F_{\infty}=\limsup _{x \rightarrow+\infty} \frac{f(x)}{x}, \\
& f_{0}=\liminf _{x \rightarrow 0^{+}} \frac{f(x)}{x}, \quad f_{\infty}=\liminf _{x \rightarrow+\infty} \frac{f(x)}{x}, \\
& C_{1}=\int_{1}^{e} G(e, s) a(s) \frac{d s}{s}, \quad C_{2}=\int_{1}^{e} g(s) G(e, s) a(s) \frac{d s}{s} .
\end{aligned}
$$

Theorem 3.1 If there exists $l \in(1, e)$ such that $g(l) f_{\infty} C_{2}>F_{0} C_{1}$ holds, for each

$$
\lambda \in\left(\left(g(l) f_{\infty} C_{2}\right)^{-1},\left(F_{0} C_{1}\right)^{-1}\right)
$$

then boundary value problem (1) has at least one positive solution. Here we impose $\left(g(l) f_{\infty} C_{2}\right)^{-1}=0$ if $f_{\infty}=+\infty$ and $\left(F_{0} C_{1}\right)^{-1}=+\infty$ if $F_{0}=0$.

Proof Let $\lambda$ satisfy (8) and $\varepsilon>0$ be such that

$$
\left(g(l)\left(f_{\infty}-\varepsilon\right) C_{2}\right)^{-1} \leq \lambda \leq\left(\left(F_{0}+\varepsilon\right) C_{1}\right)^{-1} .
$$

By the definition of $F_{0}$, we see that there exists $r_{1}>0$ such that

$$
f(x) \leq\left(F_{0}+\varepsilon\right) x, \quad \text { for } 0<x \leq r_{1} .
$$


So if $x \in \mathcal{P}$ with $\|x\|=r_{1}$, then, by (9) and (10), we have

$$
\begin{aligned}
\left\|S_{\lambda} x\right\| & \leq \lambda \int_{1}^{e} G(e, s) a(s) f(x(s)) \frac{d s}{s} \\
& \leq \lambda \int_{1}^{e} G(e, s) a(s)\left(F_{0}+\varepsilon\right) r_{1} \frac{d s}{s}=\lambda\left(F_{0}+\varepsilon\right) r_{1} C_{1} \leq r_{1}=\|x\| .
\end{aligned}
$$

Hence, if we choose $\Omega_{1}=\left\{x \in \mathcal{E}:\|x\|<r_{1}\right\}$, then we get

$$
\left\|S_{\lambda} x\right\| \leq\|x\|, \quad \text { for } x \in \mathcal{P} \cap \partial \Omega_{1} .
$$

Let $r_{3}>0$ be such that

$$
f(x) \geq\left(f_{\infty}-\varepsilon\right) x, \quad \text { for } x \geq r_{3} .
$$

If $x \in \mathcal{P}$ with $\|x\|=r_{2}=\max \left\{2 r_{1}, r_{3}\right\}$, then from (9) and (12), we obtain

$$
\begin{aligned}
\left\|S_{\lambda} x\right\| & \geq\left(S_{\lambda} x\right)(l)=\lambda \int_{1}^{e} G(e, s) a(s) f(x(s)) \frac{d s}{s} \geq \lambda \int_{1}^{e} g(l) G(e, s) a(s) f(x(s)) \frac{d s}{s} \\
& \geq \lambda \int_{1}^{e} g(l) G(e, s) a(s)\left(f_{\infty}-\varepsilon\right) x(s) \frac{d s}{s} \geq \lambda \int_{1}^{e} g(l) g(s) G(e, s) a(s)\left(f_{\infty}-\varepsilon\right)\|x\| \frac{d s}{s} \\
& =\lambda g(l) C_{2}\left(f_{\infty}-\varepsilon\right)\|x\| \geq\|x\| .
\end{aligned}
$$

Thus, if we set $\Omega_{2}=\left\{x \in \mathcal{E}:\|x\|<r_{2}\right\}$, then we get

$$
\left\|S_{\lambda} x\right\| \geq\|x\|, \quad \text { for } x \in \mathcal{P} \cap \partial \Omega_{2} .
$$

Now, from (11), (13), and Lemma 2.3, we guarantee that $S_{\lambda}$ has a fixed point $x \in \mathcal{P} \cap\left(\bar{\Omega}_{2} \backslash\right.$ $\Omega_{1}$ ) with $r_{1} \leq\|x\| \leq r_{2}$, and clearly $x$ is a positive solution of boundary value problem (1). This completes the proof of Theorem 3.1.

Theorem 3.2 If there exists $l \in(1, e)$ such that $g(l) f_{\infty} C_{2}>F_{0} C_{1}$ holds, for each

$$
\lambda \in\left(\left(g(l) f_{0} C_{2}\right)^{-1},\left(F_{\infty} C_{1}\right)^{-1}\right)
$$

then boundary value problem (1) has at least one positive solution. Here we impose $\left(g(l) f_{0} C_{2}\right)^{-1}=0$ if $f_{0}=+\infty$ and $\left(F_{\infty} C_{1}\right)^{-1}=+\infty$ if $F_{\infty}=0$.

Proof Let $\lambda$ satisfy (14) and let $\varepsilon>0$ be such that

$$
\left(g(l)\left(f_{0}-\varepsilon\right) C_{2}\right)^{-1} \leq \lambda \leq\left(\left(F_{\infty}+\varepsilon\right) C_{1}\right)^{-1}
$$

From the definition of $f_{0}$, we see that there exists $r_{1}>0$ such that

$$
f(x) \geq\left(f_{0}-\varepsilon\right) x, \quad \text { for } 0<x \leq r_{1} .
$$


Further, if $x \in \mathcal{P}$ with $\|x\|=r_{1}$, then similar to the second part of Theorem 3.1, we can obtain $\left\|S_{\lambda} x\right\| \geq\|x\|$. Thus, if we choose $\Omega_{1}=\left\{x \in \mathcal{E}:\|x\|<r_{1}\right\}$, then

$$
\left\|S_{\lambda} x\right\| \geq\|x\|, \quad \text { for } x \in P \cap \partial \Omega_{1} .
$$

Next, we may choose $R_{1}>0$ such that

$$
f(x) \leq\left(F_{\infty}+\varepsilon\right) x, \quad \text { for } x \geq R_{1} .
$$

We consider the following two cases.

Case 1. Suppose $f$ is bounded. Then there exists some $M>0$, such that

$$
f(x) \leq M, \quad \text { for } x \in(0,+\infty)
$$

We define $r_{3}=\max \left\{2 r_{1}, \lambda M C_{1}\right\}$, and $x \in \mathcal{P}$ with $\|x\|=r_{3}$, then

$$
\left\|S_{\lambda} x\right\| \leq \lambda \int_{1}^{e} G(e, s) a(s) f(x(s)) \frac{d s}{s} \leq \lambda M \int_{1}^{e} G(e, s) a(s) \frac{d s}{s}=\lambda M C_{1} \leq r_{3}=\|x\| .
$$

Hence,

$$
\left\|S_{\lambda} x\right\| \leq\|x\|, \quad \text { for } x \in \mathcal{P}_{r_{3}}=\left\{x \in \mathcal{P}:\|x\| \leq r_{3}\right\} .
$$

Case 2. Suppose $f$ is unbounded. Then there exists some $r_{4}>\max \left\{2 r_{1}, R_{1}\right\}$ such that

$$
f(x) \leq f\left(r_{4}\right), \quad \text { for } 0<x \leq r_{4}
$$

Let $x \in \mathcal{P}$ with $\|x\|=r_{4}$. Then, by (15) and (17), we have

$$
\begin{aligned}
\left\|S_{\lambda} x\right\| & \leq \lambda \int_{1}^{e} G(e, s) a(s) f(x(s)) \frac{d s}{s} \leq \lambda \int_{1}^{e} G(e, s) a(s)\left(F_{\infty}+\varepsilon\right)\|x\| \frac{d s}{s} \\
& =\lambda C_{1}\left(F_{\infty}+\varepsilon\right)\|x\| \leq\|x\| .
\end{aligned}
$$

Thus,

$$
\left\|S_{\lambda} x\right\| \leq\|x\|, \quad \text { for } x \in \mathcal{P}_{r_{4}}=\left\{x \in \mathcal{P}:\|x\| \leq r_{4}\right\} .
$$

In both Cases 1 and 2 , if we set $\Omega_{2}=\left\{x \in \mathcal{P}:\|x\|<r_{2}=\max \left\{r_{3}, r_{4}\right\}\right\}$, then

$$
\left\|S_{\lambda} x\right\| \leq\|x\|, \quad \text { for } x \in \mathcal{P} \cap \partial \Omega_{2} .
$$

It follows from (16), (18) and Lemma 2.3 that $S_{\lambda}$ has a fixed point $x \in \mathcal{P} \cap\left(\bar{\Omega}_{2} \backslash \Omega_{1}\right)$ with $r_{1} \leq\|x\| \leq r_{2}$. It is clear that $x$ is a positive solution of boundary value problem (1). The proof is complete.

Theorem 3.3 If there exist $l \in(1, e)$ and $r_{2}>r_{1}>0$ such that $g(l)>r_{1} / r_{2}$ and $f$ satisfy

$$
\min _{g(l) r_{1} \leq x \leq r_{1}} f(x) \geq \frac{r_{1}}{\lambda g(l) C_{1}}, \quad \max _{0 \leq x \leq r_{2}} f(x) \leq \frac{r_{2}}{\lambda C_{1}} .
$$

Then boundary value problem (1) has a positive solution $u$ with $r_{1} \leq\|x\| \leq r_{2}$. 
Proof Choose $\Omega_{1}=\left\{x \in \mathcal{E}:\|x\|<r_{1}\right\}$; then, for $x \in \mathcal{P} \cap \partial \Omega_{1}$, we have

$$
\begin{aligned}
\left\|S_{\lambda} x\right\| & \geq\left(S_{\lambda} x\right)(l)=\lambda \int_{1}^{e} G(e, s) a(s) f(x(s)) \frac{d s}{s} \geq \lambda \int_{1}^{e} g(l) G(e, s) a(s) f(x(s)) \frac{d s}{s} \\
& \geq \lambda g(l) C_{1} \frac{r_{1}}{\lambda g(l) C_{1}}=r_{1}=\|x\| .
\end{aligned}
$$

On the other hand, choose $\Omega_{2}=\left\{x \in \mathcal{E}:\|x\|<r_{2}\right\}$; then for $x \in \mathcal{P} \cap \partial \Omega_{2}$, we have

$$
\begin{aligned}
\left\|S_{\lambda} x\right\| & \leq \lambda \int_{1}^{e} G(e, s) a(s) f(x(s)) \frac{d s}{s} \leq \lambda \int_{1}^{e} G(e, s) a(s) \max _{0 \leq x \leq r_{2}} f(x(s)) \frac{d s}{s} \\
& \leq \lambda C_{1} \frac{r_{2}}{\lambda C_{1}}=r_{2}=\|x\| .
\end{aligned}
$$

From Lemma 2.3, boundary value problem (1) has a positive solution $x$ with $r_{1} \leq\|x\| \leq r_{2}$. The proof of Theorem 3.3 is completed.

For the remainder of this section, we will need the following assumption.

Assumption $1\left(\min _{x \in[g(l) r, r]} f(x)\right) / r>0$, where $l \in(1, e)$.

For convenience, we denote

$$
\lambda_{1}=\sup _{r>0} \frac{r}{C_{1} \max _{x \in[0, r]} f(x)}, \quad \lambda_{2}=\inf _{r>0} \frac{r}{C_{1} \min _{x \in[g(l) r, r]} f(x)} .
$$

In view of the continuity of $f(x)$ and Assumption 1 , we have $0<\lambda_{1} \leq+\infty$ and $0 \leq \lambda_{2}<$ $+\infty$.

Theorem 3.4 Suppose Assumption 1 holds. If $f_{0}=f_{\infty}=+\infty$, then boundary value problem (1) has at least two positive solutions for each $\lambda \in\left(0, \lambda_{1}\right)$.

Proof Define $\left.\varphi(r)=r / C_{1} \max _{x \in[0, r]} f(x)\right)$. By the continuity of $f(x)$ and $f_{0}=f_{\infty}=+\infty$, we see that $\varphi(r):(0,+\infty) \rightarrow(0,+\infty)$ is continuous and $\lim _{r \rightarrow 0} \varphi(r)=\lim _{r \rightarrow+\infty} \varphi(r)=0$. From (19), there exists $r_{0} \in(0,+\infty)$ such that $\varphi\left(r_{0}\right)=\sup _{r>0} \varphi(r)=\lambda_{1}$. then, for $\lambda \in\left(0, \lambda_{1}\right)$, there exist constants $c_{1}, c_{2}\left(0<c_{1}<r_{0}<c_{2}<+\infty\right)$ with $\varphi\left(c_{1}\right)=\varphi\left(c_{2}\right)=\lambda$. Thus, we have

$$
f(x) \leq \frac{c_{1}}{\lambda C_{1}}, \quad \text { for } x \in\left[0, c_{1}\right]
$$

and

$$
f(x) \leq \frac{c_{2}}{\lambda C_{1}}, \quad \text { for } x \in\left[0, c_{2}\right]
$$

On the other hand, applying the conditions $f_{0}=f_{\infty}=+\infty$, there exist constants $d_{1}, d_{2}$ $\left(0<d_{1}<c_{1}<r_{0}<c_{2}<d_{2}<+\infty\right)$ with $f(x) / x \geq 1 /\left(g^{2}(l) \lambda C_{1}\right)$, for $x \in\left(0, d_{1}\right) \cup\left(g(l) d_{2},+\infty\right)$. Then we get

$$
\min _{g(l) d_{1} \leq x \leq d_{1}} f(x) \leq \frac{d_{1}}{\lambda g(l) C_{1}}
$$


and

$$
\min _{g(l) d_{2} \leq x \leq d_{2}} f(x) \leq \frac{d_{2}}{\lambda g(l) C_{1}} .
$$

By (20) and (22), (21) and (23), combining with Theorem 3.3 and Lemma 2.3, we can complete the proof.

Corollary 3.1 Suppose Assumption 1 holds. If $f_{0}=+\infty$ or $f_{\infty}=+\infty$, then boundary value problem (1) has at least one positive solution for each $\lambda \in\left(0, \lambda_{1}\right)$.

Theorem 3.5 Suppose Assumption 1 holds. If $f_{0}=f_{\infty}=0$, then boundary value problem (1) has at least two positive solutions for each $\lambda \in\left(\lambda_{2},+\infty\right)$.

Proof Define $\psi(r)=r /\left(C_{1} \min _{x \in[g(l) r, r]} f(x)\right)$. By the continuity of $f(x)$ and $f_{0}=f_{\infty}=0$, we can easily see that $\psi(r):(0,+\infty) \rightarrow(0,+\infty)$ is continuous and $\lim _{r \rightarrow 0} \psi(r)=\lim _{r \rightarrow+\infty} \psi(r)=$ $+\infty$. From (19), there exists $r_{0} \in(0,+\infty)$ such that $\psi\left(r_{0}\right)=\sup _{r>0} \psi(r)=\lambda_{2}$, then, for $\lambda \in\left(0, \lambda_{1}\right)$, there exist constants $d_{1}, d_{2}\left(0<d_{1}<r_{0}<d_{2}<+\infty\right)$ with $\psi\left(d_{1}\right)=\psi\left(d_{2}\right)=\lambda$. Therefore,

$$
f(x) \leq \frac{d_{1}}{\lambda g(l) C_{1}}, \quad \text { for } x \in\left[g(l) d_{1}, d_{1}\right]
$$

and

$$
f(x) \leq \frac{d_{2}}{\lambda g(l) C_{1}}, \quad \text { for } x \in\left[g(l) d_{2}, d_{2}\right] .
$$

On the other hand, applying the conditions $f_{0}=0$, there exist constants $c_{1}\left(0<c_{1}<d_{1}\right)$ with $f(x) / x \leq 1 /\left(\lambda C_{1}\right)$, for $x \in\left(0, c_{1}\right)$. Then

$$
\max _{0 \leq x \leq c_{1}} f(x) \leq \frac{c_{1}}{\lambda C_{1}}
$$

In view of $f_{\infty}=0$, there exists a constant $c_{2} \in\left(d_{2},+\infty\right)$ such that $f(x) / x \leq 1 /\left(\lambda C_{1}\right)$, for $x \in\left(c_{2},+\infty\right)$. Let $M=\max _{0 \leq x \leq c_{2}} f(x), c_{2} \geq \lambda C_{1} M$. It is easily seen that

$$
\max _{0 \leq x \leq c_{2}} f(x) \leq \frac{c_{2}}{\lambda C_{1}}
$$

From (24) and (26), (25) and (27), combining with Theorem 3.3 and Lemma 2.3, we can complete the proof.

Corollary 3.2 Suppose Assumption 1 holds. If $f_{0}=0$ or $f_{\infty}=0$, then boundary value problem (1) has at least one positive solution for each $\lambda \in\left(\lambda_{2},+\infty\right)$.

By the above theorems, we can obtain the following results.

Corollary 3.3 Suppose Assumption 1 holds. If $f_{0}=+\infty, f_{\infty}=d$ or $f_{\infty}=+\infty, f_{0}=d$, then boundary value problem (1) has at least one positive solution for each $\lambda \in\left(0,\left(d C_{1}\right)^{-1}\right)$. 
Corollary 3.4 Suppose Assumption 1 holds. If $f_{0}=0, f_{\infty}=d$ or $f_{\infty}=0, f_{0}=d$, then boundary value problem (1) has at least one positive solution for each $\lambda \in\left(\left(g(l) d C_{2}\right)^{-1},+\infty\right)$.

Theorem 3.6 Suppose Assumption 1 holds. If $F_{0}<+\infty$ and $F_{\infty}<+\infty$, then there exists a $\lambda_{0}>0$ such that, for all $0<\lambda<\lambda_{0}$, boundary value problem (1) has no positive solution.

Proof Since $F_{0}<+\infty$ and $F_{\infty}<+\infty$, there exist positive numbers $m_{1}, m_{2}, r_{1}$ and $r_{2}$ such that $r_{1}<r_{2}$ and

$$
f(x) \leq m_{1} x, \quad \text { for } x \in\left[0, r_{1}\right] ; \quad f(x) \leq m_{2} x, \quad \text { for } x \in\left[r_{2},+\infty\right) .
$$

Let $\left.\left.m=\max \left\{m_{1}, m_{2}, \max _{x \in\left[r_{1}, r_{2}\right]}\right\} f(x) / x\right\}\right\}$. Then we have

$$
f(x) \leq m x, \quad \text { for } x \in[0,+\infty)
$$

Assume $x(t)$ is a positive solution of boundary value problem (1). We will show that this leads to a contradiction for $0<\lambda<\lambda_{0}:=\left(m C_{1}\right)^{-1}$. Since $S_{\lambda} x(t)=x(t)$ for $t \in[1, e]$,

$$
\|x\|=\left\|S_{\lambda} x\right\| \leq \lambda \int_{1}^{e} G(e, s) a(s) f(x(s)) \frac{d s}{s} \leq m \lambda\|x\| \int_{1}^{e} G(e, s) a(s) \frac{d s}{s}<\|x\|,
$$

which is a contradiction. Therefore, boundary value problem (1) has no positive solution. The proof is complete.

Theorem 3.7 Suppose Assumption 1 holds. If $f_{0}>0$ and $f_{\infty}>0$, then there exists a $\lambda_{0}>0$ such that, for all $\lambda>\lambda_{0}$, boundary value problem (1) has no positive solution.

Proof Since $f_{0}>0$ and $f_{\infty}>0$, there exist positive numbers $n_{1}, n_{2}, r_{1}$ and $r_{2}$ such that $r_{1}<r_{2}$ and

$$
f(u) \geq n_{1} x, \quad \text { for } x \in\left[0, r_{1}\right] ; \quad f(x) \geq n_{2} x, \quad \text { for } x \in\left[r_{2},+\infty\right) .
$$

Let $n=\min \left\{n_{1}, n_{2}, \min _{x \in\left[r_{1}, r_{2}\right]}\{f(x) / x\}\right\}$. Then we have

$$
f(x) \geq n x, \quad \text { for } x \in[0,+\infty)
$$

Assume $x(t)$ is a positive solution of boundary value problem (1). We will show that this leads to a contradiction for $\lambda>\lambda_{0}:=\left(g(l) n C_{2}\right)^{-1}$. Since $S_{\lambda} x(t)=x(t)$ for $t \in[1, e]$,

$$
\|x\|=\left\|S_{\lambda} x\right\| \geq \lambda \int_{1}^{e} g(l) G(e, s) a(s) f(x(s)) \frac{d s}{s}>\|x\|
$$

which is a contradiction. Therefore, boundary value problem (1) has no positive solution. The proof of Theorem 3.7 is completed.

\section{Some examples}

In this section, we will present some examples to illustrate the main results. 
Example 4.1 Consider the Hadamard fractional boundary value problem

$$
\left(D^{2.5} x\right)(t)+\lambda \ln t x^{\theta}(t)=0, \quad x(0)=(\delta x)(0)=(\delta x)(1)=0,1<t<e, \theta>1 .
$$

Since $\alpha=2.5$ and $a(t)=\ln t$, we have

$$
\begin{aligned}
& C_{1}=\int_{1}^{e} G(e, s) a(s) \frac{d s}{s} \leq \frac{1}{\Gamma(2.5)} \int_{1}^{e} \ln s(1-\ln s)^{0.5} \frac{d s}{s} \approx 0.2006 \\
& C_{2}=\int_{1}^{e} g(s) G(e, s) a(s) \frac{d s}{s} \geq \frac{1}{\Gamma(2.5)} \int_{1}^{e}(\ln s)^{2.5}\left((1-\ln s)^{0.5}-(1-\ln s)^{1.5}\right) \frac{d s}{s} \approx 0.1146 .
\end{aligned}
$$

Let $f(x)=x^{\theta}, \theta>1$. Then from [35], we have $F_{0}=0$ and $f_{\infty}=+\infty$. Choose $l=e^{0.5}$. Then $g\left(e^{0.5}\right)=0.5^{1.5} \approx 0.3536$. So $g(l) C_{2} f_{\infty}>F_{0} C_{1}$ holds. Thus, by Theorem 3.1, the boundary value problem (28) has a positive solution for each $\lambda \in(0,+\infty)$.

Example 4.2 Consider the Hadamard fractional boundary value problem

$$
\left(D^{2.5} x\right)(t)+\lambda \ln t x^{\vartheta}(t)=0, \quad x(0)=(\delta x)(0)=(\delta x)(1)=0,1<t<e, 0<\vartheta<1 .
$$

Since $\alpha=2.5$ and $a(t)=\ln t$, we have $C_{1} \leq 0.2006$ and $C_{2} \geq 0.1146$. Let $f(x)=x^{\vartheta}, 0<$ $\vartheta<1$. Then from [35], we have $F_{\infty}=0$ and $f_{0}=+\infty$. Choose $l=e^{0.5}$. Then $g\left(e^{0.5}\right)=0.5^{1.5} \approx$ 0.3536 . So $g(l) C_{2} f_{0}>F_{\infty} C_{1}$ holds. Thus, by Theorem 3.2, the boundary value problem (29) has a positive solution for each $\lambda \in(0,+\infty)$.

Example 4.3 Consider the Hadamard fractional boundary value problem

$$
\begin{aligned}
& \left(D^{2.5} x\right)(t)+\lambda \ln t \frac{\left(200 x^{2}(t)+x(t)\right)(2+\sin x(t))}{x(t)+1}=0, \\
& x(0)=(\delta x)(0)=(\delta x)(1)=0,1<t<e .
\end{aligned}
$$

Since $\alpha=2.5$ and $a(t)=\ln t$, we have $C_{1} \leq 0.2006$ and $C_{2} \geq 0.1146$. Let $f(x)=\left(200 x^{2}+\right.$ $x)(2+\sin x) /(x+1)$. Then from [35], we have $F_{0}=f_{0}=2, F_{\infty}=600, f_{\infty}=200$, and $2 x<f(x)<$ $600 x$.

(i) Choose $l=e^{0.5}$. Then $g\left(e^{0.5}\right)=0.5^{1.5} \approx 0.3536$. So $g(l) C_{2} f_{\infty}>F_{0} C_{1}$ holds. Thus, by Theorem 3.1 , the boundary value problem (30) has a positive solution for each $\lambda \in(0.1234,2.4925)$.

(ii) By Theorem 3.6, the boundary value problem (30) has no positive solution for all $\lambda \in(0,0.0083)$.

(iii) By Theorem 3.7, the boundary value problem (30) has no positive solution for all $\lambda \in(12.3388,+\infty)$.

\section{Conclusions}

By means of the properties of the Green function and the Guo-Krasnosel'skii fixed-point theorem on cones, we have investigated the existence and nonexistence of positive solutions for a class of boundary value problems of nonlinear Hadamard fractional differential equations with a parameter. Three examples are given to show the effectiveness of the obtained results. Furthermore, by using similarly the method in this paper, we can also obtain 
the existence and nonexistence of positive solutions for nonlinear Hadamard fractional boundary value problems as follows: $\left(D^{\alpha} x\right)(t)+\lambda a(t) f(x(t))=0, x(1)=x(e)=(\delta x)(1)=0$, $\alpha \in(2,3], t \in[1, e]$.

\section{Acknowledgements}

The authors would like to thank the anonymous referees and the editor for their constructive suggestions for improving the presentation of the paper.

\section{Competing interests}

The authors declare that they have no competing interests.

Authors' contributions

All authors contributed equally in this article. They read and approved the final manuscript.

\section{Author details}

'Faculty of General Education, Zhengzhou Technology and Business University, Zhengzhou, China. ${ }^{2}$ College of Information and Management Science, Henan Agricultural Univeristy, Zhengzhou, China.

\section{Publisher's Note}

Springer Nature remains neutral with regard to jurisdictional claims in published maps and institutional affiliations.

Received: 27 December 2017 Accepted: 8 March 2018 Published online: 15 March 2018

\section{References}

1. Agarwal, R.P., Ahmad, B., Nieto, J.J.: Fractional differential equations with nonlocal (parametric type) anti-periodic boundary conditions. Filomat 31(5), 1207-1214 (2017)

2. El-Shahed, M.: Positive solutions for boundary value problem of nonlinear fractional differential equation. Abstr. Appl. Anal. 2007, Article ID 10368 (2011)

3. Ntouyas, S.K., Tariboon, J.: Fractional integral problems for Hadamard-Caputo fractional Langevin differential inclusions. J. Appl. Math. Comput. 51, 13-33 (2016)

4. Sabatier, J., Agrawal, O.P., Tenreiro Machado, J.A.: Advances in Fractional Calculus: Theoretical Developments and Applications in Physics and Engineering. Springer, Berlin (2007)

5. Samko, S.G., Kilbas, A.A., Marichev, O.I.: Fractional Integral and Derivatives: Theory and Applications. Gordon \& Breach, Switzerland (1993)

6. Shah, K., Khalil, H., Khan, R.A.: Investigation of positive solution to a coupled system of impulsive boundary value problems for nonlinear fractional order differential equations. Chaos Solitons Fractals 77, 240-246 (2015)

7. Yakar, A., Kutlay, H.: Monotone iterative technique via initial time different coupled lower and upper solutions for fractional differential equations. Filomat 31(4), 1031-1039 (2017)

8. Wang, H., Liu, Y., Zhu, H.: Existence and stability for Hadamard $p$-type fractional functional differential equations. J. Appl. Math. Comput. 55, 549-562 (2017)

9. Zhou, Y.: Basic Theory of Fractional Differential Equations. World Scientific, Singapore (2014)

10. Zhou, Y.: Fractional Evolution Equations and Inclusions: Analysis and Control. Elsevier, New York (2016)

11. Li, Y.: Existence of positive solutions for fractional differential equation involving integral boundary conditions with p-Laplacian operator. Adv. Differ. Equ. 2017, 135 (2017)

12. Alhothuali, M.S., et al.: Nonlinear sequential fractional differential equations with nonlocal boundary conditions involving lower-order fractional derivatives. Adv. Differ. Equ. 2017, 104 (2017)

13. Ahmadkhanlu, A.: Existence and uniqueness results for a class of fractional differential equations with an integral fractional boundary condition. Filomat 31(5), 1241-1249 (2017)

14. Cabada, A., Wang, G.: Positive solutions of nonlinear fractional differential equations with integral boundary value conditions. J. Math. Anal. Appl. 389, 403-411 (2012)

15. Ahmad, B., Nieto, J.J.: Existence results for nonlinear boundary value problems of fractional integrodifferential equations with integral boundary conditions. Bound. Value Probl. 2009, 708576 (2009)

16. Yang, W.: Positive solutions for nonlinear Caputo fractional differential equations with integral boundary conditions. J. Appl. Math. Comput. 44, 39-59 (2014)

17. Henderson, J., Luca, R.: Boundary Value Problems for Systems of Differential, Difference and Fractional Equations: Positive Solutions. Elsevier, Amsterdam (2015)

18. Liu, Y., et al:: Multiple positive solutions for a coupled system of fractional multi-point BVP with p-Laplacian operator. Adv. Differ. Equ. 2017, 168 (2017)

19. Ahmad, B., Ntouyas, S.K.: Initial-value problems for hybrid Hadamard fractional differential equations. Electron. J. Differ. Equ. 2014, 161 (2014)

20. Alsaedi, A., Ntouyas, S.K., Ahmad, B., Hobiny, A.: Nonlinear Hadamard fractional differential equations with Hadamard type nonlocal non-conserved conditions. Adv. Differ. Equ. 2015, 285 (2015)

21. Li, M., Wang, J.: Stable manifolds results for planar Hadamard fractional differential equations. J. Appl. Math. Comput. $55,645-668(2017)$

22. Thiramanus, P., Ntouyas, S.K., Tariboon, J.: Positive solutions for Hadamard fractional differential equations on infinite domain. Adv. Differ. Equ. 2016, 83 (2016)

23. Yukunthorn, W., Suantai, S., Ntouyas, S.K., Tariboon, J.: Boundary value problems for impulsive multi-order Hadamard fractional differential equations. Bound. Value Probl. 2015, 148 (2015) 
24. Yang, W., Qin, Y.: Positive solutions for nonlinear Hadamard fractional differential equations with integral boundary conditions. ScienceAisa 43, 201-206 (2017)

25. Ahmad, B., Ntouyas, S.K.: On Hadamard fractional integro-differential boundary value problems. J. Appl. Math. Comput. 47, 119-131 (2015)

26. Ahmad, B., Ntouyas, S.K.: A fully Hadamard type integral boundary value problem of a coupled system of fractional differential equations. Fract. Calc. Appl. Anal. 17, 348-360 (2014)

27. Ahmad, B., Ntouyas, S.K., Alsaedi, A.: New results for boundary value problems of Hadamard-type fractional differential inclusions and integral boundary conditions. Bound. Value Probl. 2013, 275 (2013)

28. Aljoudi, S., Ahmad, B., Niet, J.J., Alsaedi, A.: A coupled system of Hadamard type sequential fractional differential equations with coupled strip conditions. Chaos Solitons Fractals 91, 39-46 (2016)

29. Li, M., Wang, J.: Analysis of nonlinear Hadamard fractional differential equations via properties of Mittag-Leffler functions. J. Appl. Math. Comput. 51, 487-508 (2016)

30. Yang, W.: Positive solutions for singular Hadamard fractional differential system with four-point coupled boundary conditions. J. Appl. Math. Comput. 49, 357-381 (2015)

31. Yang, W.: Positive solutions for singular coupled integral boundary value problems of nonlinear Hadamard fractional differential equations. J. Nonlinear Sci. Appl. 8, 110-129 (2015)

32. Kilbas, A.A., Srivastava, H.M., Trujillo, J.J.: Theory and Applications of Fractional Differential Equations. Elsevier, Amsterdam (2006)

33. Guo, D.J., Lakshmikantham, V:: Nonlinear Problems in Abstract Cones. Academic Press, Boston (1988)

34. Krasnosel'skii, M.A.: Positive Solutions of Operator Equations. Noordhoff, Groningen (1964)

35. Zhao, Y., et al.: Positive solutions to boundary value problems of nonlinear fractional differential equations. Abstr. Appl. Anal. 2011, Article ID 390543 (2011)

\section{Submit your manuscript to a SpringerOpen ${ }^{\circ}$ journal and benefit from:}

- Convenient online submission

- Rigorous peer review

- Open access: articles freely available online

- High visibility within the field

- Retaining the copyright to your article

Submit your next manuscript at $\boldsymbol{\nabla}$ springeropen.com 